\title{
0401 EXAMINING THE VALIDITY OF EMERGENCY DEPARTMENT TEXTUAL DATA FOR INJURY SURVEILLANCE PURPOSES
}

D Scott*, R Barker, G Nand, L Horth, P Smith, K McKenzie Correspondence: Queensland Injury Surveillance Unit (OISU)/National Centre for Health Information Research and Training (NCHIRT), Mater Hospital Level 1 Whitty Building, Raymond Terrace, South Brisbane, OLD 4101 NCHIRT, School of Public Health, Queensland University of Technology, Victoria Park Road, Kelvin Grove OLD, 4101, Australia

10.1136/ip.2010.029215.401

Injury surveillance data provides the evidence base to inform injury prevention programme and policy development. The Queensland Injury Surveillance Unit (QISU) collects data on emergency department (ED) injury presentations at Queensland hospitals. When a patient presents for treatment of an injury, the triage nurse activates an injury surveillance screen and assigns codes for intent, place, part of place, activity, subtype of activity, major injury factor, mechanism and external cause of injury. In busy ED situations this can be time consuming, 3-5 min/record. OISU investigated the possibility of reducing the number of coded fields and having QISU coders assign the remaining codes based on the triage presenting problem text field. 500 records were randomly sampled from the database. Two experienced OISU coders were blinded to the codes assigned at triage and recoded the records using only the presenting problem text descriptor. The quality of the information contained within the text descriptor was assessed by recording what variables were able to be coded from the text descriptor. Concordance between data coded by triage nurses and OISU coders was compared. There was variability in the concordance of codes and the quality of the text descriptor between facilities, injury type and patient groups. This information will be used to streamline data collection by reducing the number of variables coded by the triage nurse and demonstrates the utility of textual data for injury surveillance purposes. 